\title{
VORDERASIATISCHE BIBLIOTHEK
}

\section{DIE \\ EL-AMARNA-TAFELN}

BEARBEITET

vON

J. A. KNUDTZON

ERSTER TEIL: TEXTE

LEIPZIG

J. C. HINRICHS' SCHE BUCHHANDLUNG 1910 
In der Reihenfolge des Erscheinens der Vorderasiatischen Bibliothek

2. Stück 


\title{
DIE EL-AMARNA-TAFELN
}

\author{
bearbeitet von J. A. Knudtzon
}

Hiervon erschien soeben:

Erste Hälfte (= Lieferung I-6)

zum Subskriptionspreis (je $M 2.50 \Rightarrow$ M 15

Einleitung . . . . . . . . . . . . . . . S. 1-59 Umschrift und Übersetzung der Tafeln . . . . SS. $60 \mathrm{ff}$. Erläuterungen.

Liste der Originale, geordnet nach ihren Besitzern. Undeutliche oder fragliche Zeichen in den Originalen.

Sachliche Anmerkungen.

Namen- und Wörterverzeichnisse.

\section{Ubersicht der Briefe:}

Zbdi-Aširte (bezw. Ašratu) an den König. Nr. 60. 61.

" " an Pabanate. Nr. 62.

Ebdi-Ašta(r)ti an den König. Nr. 63. 64; auch Nr. 66?

bdibiba-von Jerusalem an den König. 6 oder 7 Briefe: Nr. 285-29o; vielleicht auch $\mathrm{Nr}$. $29 \mathrm{r}$.

Ebdimilki von Šashimi an den König. Nr. 203.

Abdi-na _ _ - an den König. Nr. 229.

Abdi-tirši von Hazur an den König. Nr. 228.

Abimilki von Tyrus an den König. ro Briefe: Nr. 146-155.

Addu- . . . . an den König. Nr. 249. 250.

Addu-dâni an den König. Nr. 292; etwa auch Nr. 293-295.

Addu-nirari an den König von Ảgypten. Nr. 5 r.

Adra-Aštarti (= Abdi-A.?) an den König. Nr. 65 .

Ägyptens König an den Fürsten von Ammia (?). Nr. 99.

" " (bezw. Hof) an den Fürsten von Amurra. Nr. 162; wohl auch 163.

" "(bezw. Hof) an Etakkama? Nr. Igo.

$A\left[\xi_{-}-\right]_{\text {an den König. Nr. } 217 .}$

Akizzi von Kațna an Amenophis III. Nr. 52- 55 .

Alasia-König an den König von Ägypten. 7 Briefe: Nr. 33-39.

Alasia-rabiș an den rabiș von Ägypten. Nr. 40.

Amajaše an den König. Nr. 202.

Amenophis III an Kadašman-Harbe. Nr. 1. 5.

" " an Tarhundaraba, meistens in der Arzawa-Sprache. Nr. 31.

Ammunira von Bêrût an den König. Nr. 141-143.

Ara[ba]ttu - von Kumidi an den König. Nr. 198.

Artamanja von Ziribašani an den König. Nr. 201. 
Erzawa-Brief Nr. 2 (vgl. Amenophis III an Tarbundaraba). Nr. 32

rzawija von Rubiza an den König. Nr. 191. 192.

Sur-uballit an Amenophis IE. Nr. 15. 16.

Eziri an Dûdu. Nr. 158. 164.

" " an Hai. Nr. 166. 167(?).

", an den König. 7 Briefe: Nr. 156. 157. 159-161. 165. 168.

ziri-Sohn an einen hohen ägypt. Beamten (Hai?). Nr. 169.

Ziri-Sohn(?) an den König. Nr. 17r.

Babylonische (vgl. S. 20) Prinzessin an ihren Herrn. Nr. 12.

Baduzan[a] an den König. Nr. 239.

Bajadi an einen Großen. Nr. 238.

" " an den König. Nr. 237.

Baiawa an den König. Nr. 215. 216.

Balumiir (etwa $=$ Ba'lumibir) an den König. Nr. 260.

Ba‘aluia und Battiilu an den Kŏnig. Nr. 170.

Baclat- ..... an den König. Nr. 273. 274.

$B a^{c} l u$-mihir an den König. Nr. 257-259.

Bieri von Hašabu an den König. Nr. 174.

Biridija von Megiddo an den König. 5 Briefe: Nr. 242-246.

Biridija oder Jašdata an den König. Nr. 247.

Burraburiaš an Amenophis III(?). Nr. 6.

$" n$ an Amenophis IV. 5 Briefe: Nr. 7-1r.

Cölesyrische (vgl. S. 57 oben) verstümmelte Briefe an den König. Nr. I76.

179-181. 188; etwa auch Nr. 172 (ein Verzeichnis?), 173 u. 199.

Dagantakala an den König. Nr. 317. 318.

Dašru an den König. Nr. 26r. 262.

„Diener" an den Kőnig. Nr. 200.

Dijate an den Kőnig. Nr. 193.

$E$ bi od. [P]abi an einen Großen. Nr. 333.

$\operatorname{En}[g] \hat{a}[t] a$ an den König. Nr. 223.

Etakkama an den König. Nr. 189.

Gubbu-Fürst an den König. Nr. 205.

Gubla (Stadt) siehe unter Ilirabib.

Gubla-Mann an den König. Nr. 94.

" $"$ an einen hohen ägypt. Beamten oder den König. Nr. 1or.

Hatti(?)-König (vgl. Subbiluliuma) an den Kőnig von Ägypten. Nr. 42.

Hazur-König (vgl. Abdi-tirši) an den König. Nr. 227.

Hibija an einen Großen. Nr. 178.

Hiziri an den König. Nr. 336. 337.

Iabniilu von Lakiš an den König. Nr. 328.

Ia[b]tiri an den König. Nr. 296.

Iabzibada an den König. Nr. 275. 276.

Iama an den König. : Nr. 230.

Jamiuta von Guddašuna an den König. Nr. r77.

Iapah-Addi an Ianbamu. Nr. 98.

Iapabi von Gazri an den König. Nr. 297-299; wahrscheinlich auch 300. 
Iappa[b-Addi] an Šumu-badi. Nr. 97.

Ja[šd]ata an den König. Nr. 248; siehe auch Biridija.

$n$ dâja von Hazi an den König. Nr. 175.

Ilirạbih und die Stadt Gubla an den König. Nr. 139.140.

Ipte _ _ an den König. Nr. 207.

Irḳata (Stadt) an den König. Nr. 100.

Kadašman-Harbe an Amenophis III. Nr. 2-4.

Labaja an den König. Nr. 252-254.

Majarzana von Hazi an den Kŏnig. Nr. 185. 186.

Milkili an den König. 5 Briefe: Nr. 267-27r.

$[M]$ istu _ _ - an den König. Nr. 45.

Mittelpăāstinensische(?) unvollständige Briefe. Nr. 25r an einen hohen: ăgypt. Beamten; Nr. 263 wohl an den König.

Mut-Batlu an Ianbamu. Nr. 256.

", an den König. Nr.. 255.

Namiawaza an den König. Nr. r94-197.

Naziba-Fürst an den Kőnig. Nr. 206.

Nordpalästinensische (?; vgl. S. 58) verstümmelte Briefe an den König. Nr. 214. 218. 219. 23r; etwa auch Nr. 208. 236. 240.

Nordsyrischer(?) König an den König von Ägypten. Nr. 43.

", , an die Könige Kanaans. Nr. 30 .

Nordsyrische (so m. E.) verstümmelte Briefe: a) Nr. 46 und 47 , beide an den König, mit dem Brief von [M] istu _ - - (Nr. 45) eng verwandt; b) Nr. 56 und 57, sich eng an die Briefe Akizzis (Nr. 52-55)

Nukurturwa von(?) $[Z]$ ] nu an den König. Nr. 220 . [anschließend. "Oberst" an" Rib-Addi. Nr. 96.

[P]abi siehe Ebi.

Phönizischer (?) verstümmelter Brief an den König. Nr.67.

Pu-Ba'lu von Jurșa an den König. Nr. 314. 315.

", an den König und an Šǎ̧̌sibašiba. Nr. 316.

Kana-Fürst an den König. Nr. 204.

[Kat]iłutišupa an den König(?). Nr. 58.

Rib-Addi an Amanappa. 6 Briefe: Nr. 73. 77. 82. 86. 87. 93.

$" n$ an einen hohen ägypt. Beamten. Nr.69.1 13 (vielleicht an d. Kōnig).

$n$, an einen Großen. Nr. 95.

" " an Haia(?). Nr. $7 \mathbf{r}$.

", an [Ianba]m[u]. Nr. ro2.

$" n$ an den König. 53 Briefe: Nr. 68. 70. 72. 74-76. 78-81. 83-85. 88-92. 103-r12. $\mathrm{II}_{4}-\mathrm{r} 38$.

Rușmania von Saruna an den König. Nr. 241.

Šama-Adda an den König. Nr. 49.

Samn-Adda von Sambuna an den König. Nr. 225.

Šatija an den Kōnig. Nr. 187 .

Sipti-Ballu an den Konig. Nr. 330. 331; wohl auch 332:

Siptur[i _] an den König. Nr. 226.

Subandu an den Kornig. 6 Briefe: Nr. 301-306. 
Subbiluliuma an Hori[a]. Nr. 4r.

Šum-add $[a]$ an den König. Nr. 224.

Sum _ _ - an den König. Nr. 272.

Sutarna von Mušihuna an den König. Nr. $18 \dot{2}=184$.

Šuwardata an den König. 7 Briefe: Nr. 278-284.

Südpalästinensische verstümmelte Briefe an den König (so wenigstens die meisten). Nr. 277 (von Iabzibada?), Nr. 307-313 (einige von diesen etwa von Subandu) und Nr. 327 (von Widia?).

Tagi an den König. Nr. 264. 265; auch 266?

"Tochter von _ -“ an ihre Herrin $B\left[i{ }_{-}-\right]$(vgl. S. 2r oben). Nr. 50.

Tunips Einwohner an den König. Nr. 59.

Tušratta an Amenophis III. 7 Briefe: Nr. 17-21. 23. 24.

$"$, an Amenophis IV. Nr. 27-29.

" " an Teie, Witwe Amenophis' III. Nr. 26.

Widia von Askalon an den König. 7 Briefe: Nr. 320-326

Wiktazu an den König. Nr. 22x. 222.

Zatatna von Akko an den König. Nr. 233. 234.

$Z[i$ _ - ] an den König. Nr. 338.

$\mathrm{Z} \hat{\mathrm{i}}[k] a r$, ein nordsyr. „Königssohn", an den König von Ägypten. Nr. 44. [Z]imrid[a] an einen hohen (aggyt.) Beamten. Nr. 145.

Zimriddi von Șidon an den König. Nr. 144.

Zimridi von Lakiš an den Kônig. Nr. 329.

Zišami[mi] an Amenophis IV?. Nr. 2 ro.

Zišamimi an den König. Nr. 209.

Zitatna an den König. Nr. 235.

Zitrijara an den Kōnig. Nr. 211-213.

Zurašar von $A[\xi]$ tirumna an den König. Nr. 319. 